\title{
ON THE EXISTENCE OF UNIFORMLY CONSISTENT ESTIMATES ${ }^{1}$
}

\author{
YANNIS G. YATRACOS
}

AbStraCt. Let $\mathscr{M}$ be a family of probability measures on $(\mathscr{X}, \mathscr{A})$ and $U$ the uniform structure defined by vicinities of the form

$$
\left\{(P, Q): \sup _{1 \leqslant i \leqslant K}\left|P^{n}\left(A_{i, n}\right)-Q^{n}\left(A_{i, n}\right)\right|<\varepsilon\right\},
$$

where $P^{n}$ is the product measure on $\left(\mathscr{X}^{n}, \mathscr{A}^{n}\right), A_{i, n} \in \mathscr{A}^{n}, \varepsilon>0, n \wedge K \geqslant 1$. Let $\phi^{*}:(\mathscr{M}, U) \rightarrow\left(\phi^{*}(\mathscr{M}), d\right)$, where

$$
d\left(\phi^{*}(P), \phi^{*}(Q)\right)=\|P-Q\|_{I_{1}}=2 \sup _{A \in \mathscr{A}}|P(A)-Q(A)| .
$$

We consider the case where the space of measures $M$ is $L_{1}$ separable and relate the existence of uniformly consistent estimates for $\phi^{*}(P)$ with uniform continuity of $\phi^{*}$ and $L_{1}$-total boundedness of $M$.

1. Introduction, notation and definitions. Let $(\mathscr{X}, \mathscr{A})$ be a space with a $\sigma$-field, let $\mathscr{M}$ be a family of probability measures on $\mathscr{A},\left(\mathscr{X}^{n}, \mathscr{A}^{n}\right)$ the $n$th product space and $\sigma$-field, and let $X_{1}, \ldots, X_{n}$ be independent identically distributed observations according to some measure $P \in \mathscr{M}, P^{n}$ being the $n$th product measure. Let $\Theta$ be a topological space which is homeomorphic to a subset of $[0,1]^{\infty}, h$ being the homeomorphism, and let $\rho$ be a metric on $[0,1]^{\infty}$ of the form

$$
\rho(x, y)=\sum_{m=1}^{\infty} 2^{-m}\left|x_{m}-y_{m}\right|,
$$

where $x_{m}, y_{m}$ are coordinates of $x, y$ respectively. Let $\phi^{*}: P \rightarrow \phi^{*}(P)$ be a function defined on $\mathscr{M}$ with values in $\Theta$, and $\phi=h \circ \phi^{*}, \phi: \mathscr{M} \rightarrow\left([0,1]^{\infty}, \rho\right)$.

LeCam and Schwartz [1960] gave necessary and sufficient conditions for the existence of uniformly consistent estimates of $\phi(P)$ in terms of the uniform continuity of $\phi$ with respect to a uniform structure $U=\bigcup_{n} U_{n}$, where each $U_{n}$ consists of vicinities of the form $\left\{(P, Q): \sup _{1 \leqslant j \leqslant l}\left|\int f_{j} d P^{n}-\int f_{j} d Q^{n}\right|<1\right.$ for $f_{1}, \ldots, f_{l}$ bounded measurable functions on the product space $\left.\left(\mathscr{X}^{n}, \mathscr{A}^{n}\right)\right\}$. The same uniform structure has been used by Pfanzagl [1968] and Moché [1977]. Under the above set-up we will explain why it is natural to consider the uniform structure $U$ and we

Received by the editors May 11, 1984 and, in revised form, September 17, 1984.

1980 Mathematics Subject Classification. Primary 62G05.

Key words and phrases. Uniformly consistent estimation of a functional of a measure, $L_{1}$ total boundedness of the space of measures, minimum distance estimation.

${ }^{1}$ This research was partially supported by the Hellenic Government and UNESCO. In 1982 it was also partially supported by National Science Foundation Grant MCS80-02698. 
will offer a theorem on the existence of estimates. Relaxing the condition of homeomorphism of $\Theta$ with a subset of $[0,1]^{\infty}$ and assuming only that $\left(\phi^{*}(\mathscr{M}), d\right)$ is separable when metrized with the total variation $\left(L_{1}\right)$ norm between the measures, i.e. $d\left(\phi^{*}(P), \phi^{*}(Q)\right)=\|P-Q\|_{L_{1}}=2 \sup \{|P(A)-Q(A)| ; A \in \mathscr{A}\}$, we offer a theorem of the same type in the form of equivalent propositions.

DEFINITION. We shall say $\phi$ is uniformly consistently estimable if there is a sequence $T_{n}$ of measurable functions from $\left(\mathscr{X}^{n}, \mathscr{A}^{n}\right)$ to $\left([0,1]^{\infty}, \rho\right)$ such that for every $\varepsilon>0$, $\sup \left\{P^{n}\left[\rho\left(T_{n}, \phi(P)\right)>\varepsilon\right] ; P \in \mathscr{M}\right\} \rightarrow 0$ as $n$ tends to infinity.

REMARK. One can easily see that since $\rho$ is bounded, the above definition is equivalent to $\sup \left\{E_{P^{n}} \rho\left(T_{n}, \phi(P)\right): P \in \mathscr{M}\right\} \rightarrow 0$ as $n$ tends to infinity.

Recall from topology the following

Definition. Let $(Y, d)$ be a metric space with a metric $d$. We say $Y$ is $d$-totally bounded if for every $\varepsilon>0$ there are $y_{1}, \ldots, y_{n(\varepsilon)}$ elements of $Y$ such that

$$
Y=\bigcup_{i=1}^{n(\varepsilon)}\left\{y: d\left(y, y_{i}\right)<\varepsilon\right\}
$$

In our effort to relax the hypothesis of homeomorphism of $\Theta$ with $[0,1]^{\infty}$ our estimator will take values in an abstract space. Terminology and results under this set-up concerning measurability (strong and weak), convergence (almost sure and almost uniform) and Bochner integration can be found in Hille and Phillips [1957, $\S \S 3.5$ and 3.7]. For the notion of uniform structure the reader is referred to Choquet [1969, Chapter 2, §5]. For a deep insight on consistency questions, we suggest the remarkable paper of Kraft [1955].

\section{A note on the LeCam-Schwartz set-up.}

Lemma 1. Assume $\phi$ is uniformly consistently estimable by $T_{n}$. Then for every $\varepsilon>0$ there exist positive integers $n=n(\varepsilon), K=K(\varepsilon)$ and $l=l(\varepsilon)$, and measurable sets $B_{1}^{\prime \prime}, \ldots, B_{l}^{\prime \prime}$ of the nth product $\sigma$-field $\mathscr{A}^{n}$ such that

$$
\rho(\phi(P), \phi(Q)) \leqslant \varepsilon+K \cdot \sup \left\{\left|P^{n}\left(B_{j}^{n}\right)-Q^{n}\left(B_{j}^{n}\right)\right| ; 1 \leqslant j \leqslant l(\varepsilon)\right\} .
$$

Proof. We will use the triangular inequality and then Jensen's inequality,

$$
\begin{aligned}
\rho(\phi(P), \phi(Q)) & \leqslant \rho\left(\phi(P), E_{P^{n}} T_{n}\right)+\rho\left(E_{P^{n}} T_{n}, E_{Q^{n}} T_{n}\right)+\rho\left(E_{Q^{n}} T_{n}, \phi(Q)\right) \\
& \leqslant E_{P^{n}} \rho\left(\phi(P), T_{n}\right)+\rho\left(E_{P^{n}} T_{n}, E_{Q^{n}} T_{n}\right)+E_{Q^{n}} \rho\left(T_{n}, \phi(Q)\right) \\
& \leqslant 2 \cdot \frac{\varepsilon}{4}+\rho\left(E_{P^{n}} T_{n}, E_{Q^{n}} T_{n}\right)
\end{aligned}
$$

for every $n \geqslant n(\varepsilon)$ by uniform convergence of $T_{n}$ to $\phi(P)$. For the rest of the proof let $n=n(\varepsilon)$.

Consider

$$
\rho\left(E_{P^{n}} T_{n}, E_{Q^{n}} T_{n}\right)=\sum_{m=1}^{\infty} 2^{-m}\left|E_{P^{n}}\left(T_{n}\right)_{m}-E_{Q^{n}}\left(T_{n}\right)_{m}\right|
$$


where $\left(T_{n}\right)_{m}$ are the coordinates of $T_{n}, 1 \leqslant m<\infty$. By assumption $\mid E_{P^{n}}\left(T_{n}\right)_{m}-$ $E_{Q^{n}}\left(T_{n}\right)_{m} \mid \leqslant 2$, so there is $m_{0}=m_{0}(\varepsilon)>0$ such that

$$
\sum_{m=m_{0}+1}^{\infty} 2^{-m}\left|E_{P^{n}}\left(T_{n}\right)_{m}-E_{Q^{n}}\left(T_{n}\right)_{m}\right| \leqslant \sum_{m=m_{0}+1}^{\infty} 2^{-m+1}<\frac{\varepsilon}{4} .
$$

So now (2) becomes

$$
\rho\left(E_{P^{n}} T_{n}, E_{Q^{n}} T_{n}\right) \leqslant \frac{\varepsilon}{4}+\sum_{m=1}^{m_{0}} 2^{-m}\left|E_{P^{n}}\left(T_{n}\right)_{m}-E_{Q^{n}}\left(T_{n}\right)_{m}\right| .
$$

Consider now $\left(T_{n}\right)_{m}$ for some $m \in\left\{1, \ldots, m_{0}\right\}$. Since $0 \leqslant\left(T_{n}\right)_{m} \leqslant 1$ for $\varepsilon / 8$ there exists a simple function of the form $\sum_{i=1}^{l_{m}(\varepsilon)} a_{i, m} I_{A_{i, m}^{n}}$ such that

$$
\sup \left\{\left|\left(T_{n}\left(x_{1}, \ldots, x_{n}\right)\right)_{m}-\sum_{i=1}^{I_{m}(\varepsilon)} a_{i, m} I_{A_{i, m}^{n}}\right| ;\left(x_{1}, \ldots, x_{n}\right) \in \mathscr{X}^{n}\right\}<\frac{\varepsilon}{8} \text {. }
$$

The $m$ th term of the sum in (3) becomes

$$
\begin{aligned}
& \left|E_{P^{n}}\left(T_{n}\right)_{m}-E_{Q^{n}}\left(T_{n}\right)_{m}\right| \leqslant \frac{2 \varepsilon}{8}+\sum_{i=1}^{I_{m}(\varepsilon)} a_{i, m}\left|P^{n}\left(A_{i, m}^{n}\right)-Q^{n}\left(A_{i, m}^{n}\right)\right| \\
& \leqslant \frac{\varepsilon}{4}+\left(\sum_{i=1}^{I_{m}(\varepsilon)}\left|a_{i, m}\right|\right) \cdot \sup \left\{\left|P^{n}\left(A_{i, m}^{n}\right)-Q^{n}\left(A_{i, m}^{n}\right)\right| ; 1 \leqslant i \leqslant l_{m}(\varepsilon)\right\} .
\end{aligned}
$$

Letting $K_{m}(\varepsilon)=\sum_{i=1}^{l_{m}(\varepsilon)}\left|a_{i, m}\right|$ and repeating the same argument for all $m \in$ $\left\{1, \ldots, m_{0}\right\}$ in $(3)$ we get

$$
\begin{aligned}
& \rho\left(E_{P^{n}} T_{n}, E_{Q^{n}} T_{n}\right) \leqslant \frac{\varepsilon}{4}+\sum_{m=1}^{m_{0}} 2^{-m} \cdot \frac{\varepsilon}{4} \\
& +\sum_{m=1}^{m_{0}} 2^{-m} \cdot K_{m}(\varepsilon) \cdot \sup \left\{\left|P^{n}\left(A_{i, m}^{n}\right)-Q^{n}\left(A_{i, m}^{n}\right)\right| ; 1 \leqslant i \leqslant l_{m}(\varepsilon)\right\} \\
& \leqslant \frac{\varepsilon}{2}+\left(\sum_{m=1}^{m_{0}} 2^{-m} K_{m}(\varepsilon)\right) \cdot \sup \left\{\left|P^{n}\left(B_{j}^{n}\right)-Q^{n}\left(B_{j}^{n}\right)\right| ; 1 \leqslant j \leqslant \sum_{m=1}^{m_{0}} l_{m}(\varepsilon) ;\right. \\
& \left.B_{j}^{n} \in\left\{A_{i, m}^{n}, 1 \leqslant m \leqslant m_{0}, 1 \leqslant i \leqslant l_{m}(\varepsilon)\right\}\right\} .
\end{aligned}
$$

By letting $K(\varepsilon)=\sum_{m=1}^{m_{0}} 2^{-m} K_{m}(\varepsilon), l(\varepsilon)=\sum_{m=1}^{m_{0}} l_{m}(\varepsilon)$ and replacing in (1) we get

$$
\rho(\phi(P), \phi(Q)) \leqslant \varepsilon+K \cdot \sup \left\{\left|P^{n}\left(B_{j}^{n}\right)-Q^{n}\left(B_{j}^{n}\right)\right| ; 1 \leqslant j \leqslant l\right\} .
$$

Q.E.D.

The above lemma shows that giving $\mathscr{M}$ the uniform structure consisting of vicinities of the form $\left\{(P, Q)\right.$ : $\left.\sup \left\{\left|P^{n}\left(A_{i}^{n}\right)-Q^{n}\left(A_{i}^{n}\right)\right| ; 1 \leqslant i \leqslant l\right\}<\varepsilon\right\}$ for $l \in N^{+}$, $n \in N^{+}, \varepsilon>0$, is the natural way for associating uniform convergence in probability of $T_{n}$ with the uniform continuity of $\phi: \mathscr{M} \rightarrow\left([0,1]^{\infty}, \rho\right)$.

It is now easy to see that these vicinities give rise to the same uniform structure $U$ as those in the introduction. 
We offer the following

Lemma 2. Let $\phi:(\mathscr{M}, U) \rightarrow\left([0,1]^{\infty}, \rho\right)$. The following are equivalent:

(1) $\phi$ is uniformly continuous.

(2) For every $\varepsilon>0$ there exists $K(\varepsilon), m(\varepsilon), n(\varepsilon)$ all positive, and $B_{1}^{m(\varepsilon)}, \ldots, B_{n(\varepsilon)}^{m(\varepsilon)}$ elements of the $m(\varepsilon)$-product $\sigma$-field $\mathscr{A}^{m(\varepsilon)}$ such that

$\rho(\phi(P), \phi(Q)) \leqslant \varepsilon+K(\varepsilon) \cdot \sup \left\{\left|P^{m(\varepsilon)}\left(B_{i}^{m(\varepsilon)}\right)-Q^{m(\varepsilon)}\left(B_{i}^{m(\varepsilon)}\right)\right|: 1 \leqslant i \leqslant n(\varepsilon)\right\}$.

Proof. (2) $\Rightarrow$ (1). Obvious.

$(1) \Rightarrow(2)$. Since $\phi$ is uniformly continuous, for every $\varepsilon>0$ there is $\delta(\varepsilon)>0$, $m=m(\varepsilon)>0, n=n(\varepsilon)>0$ and sets $B_{1}^{m}, \ldots, B_{n}^{m}$ in $\mathscr{A}^{m}$ such that if

$$
\sup \left\{\left|P^{m}\left(B_{i}^{m}\right)-Q^{m}\left(B_{i}^{m}\right)\right| ; 1 \leqslant i \leqslant n\right\}<\delta(\varepsilon),
$$

then $\rho(\phi(P), \phi(Q))<\varepsilon$.

From this, for every $P, Q$ such that $\rho(\phi(P), \phi(Q)) \geqslant \varepsilon$ it is necessary that $\sup \left\{\left|P^{m}\left(B_{i}^{m}\right)-Q^{m}\left(B_{i}^{m}\right)\right| ; 1 \leqslant i \leqslant n\right\} \geqslant \delta(\varepsilon)$.

Going to the statement it is required to prove: Note that for every $\varepsilon>0$ either $\rho(\phi(P), \phi(Q))<\varepsilon$ or $\rho(\phi(P), \phi(Q)) \geqslant \varepsilon$. For the second case we have then

$$
\begin{gathered}
\rho(\phi(P), \phi(Q)) \leqslant \rho(\phi(P), \phi(Q)) \cdot\left[\operatorname { i n f } \left\{\sup \left\{\left|P^{m}\left(B_{i}^{m}\right)-Q^{m}\left(B_{i}^{m}\right)\right| ; 1 \leqslant i \leqslant n\right\} ;\right.\right. \\
P, Q: \rho(\phi(P), \phi(Q))>\varepsilon\}]^{-1} \\
\cdot \sup \left\{\left|P^{m}\left(B_{i}^{m}\right)-Q^{m}\left(B_{i}^{m}\right)\right| ; 1 \leqslant i \leqslant n\right\} \\
\leqslant 2[\delta(\varepsilon)]^{-1} \sup \left\{\left|P^{m}\left(B_{i}^{m}\right)-Q^{m}\left(B_{i}^{m}\right)\right| ; 1 \leqslant i \leqslant n\right\} .
\end{gathered}
$$

Let $2[\delta(\varepsilon)]^{-1}=K(\varepsilon)$. So finally for all $P, Q$

$$
\rho(\phi(P), \phi(Q)) \leqslant \varepsilon+K(\varepsilon) \cdot \sup \left\{\left|P^{m}\left(B_{i}^{m}\right)-Q^{m}\left(B_{i}^{m}\right)\right| ; 1 \leqslant i \leqslant n\right\} .
$$

We offer

THEOREM 1. Let $\phi:(\mathscr{M}, U) \rightarrow\left([0,1]^{\infty}, \rho\right)$ with $U$ defined as above. The following statements are equivalent:

(1) $\phi$ is uniformly continuous.

(2) There exists a uniformly consistent estimator of $\phi(P)$.

Proof. (1) $\Rightarrow(2)$. Given in LeCam and Schwartz [1960, Theorem 1].

$(2) \Rightarrow(1)$. Corollary of Lemmas 1 and 2.

3. The main result. Consider now the case $\phi^{*}:(\mathscr{M}, U) \rightarrow\left(\phi^{*}(\mathscr{M}),\|\cdot\|_{L_{1}}\right)$, as in the introduction, with $\phi^{*}(\mathscr{M})$ separable when metrized with the $L_{1}$-distance between measures. We will give our result in a series of lemmas.

LEMMA 3. The uniform structure $U$ is precompact (in the sense that for any vicinity of the form $\left\{(P, Q): \sup \left\{\left|P^{m}\left(A_{i}^{m}\right)-Q^{m}\left(A_{i}^{m}\right)\right| ; 1 \leqslant i \leqslant n\right\}<\varepsilon\right\}$ there exist $P_{1}, \ldots, P_{l}$ in $\mathscr{M}$ such that $\left.\mathscr{M}=\bigcup_{k=1}^{l}\left\{P: \sup \left\{\left|P^{m}\left(A_{i}^{m}\right)-P_{k}^{m}\left(A_{i}^{m}\right)\right| ; 1 \leqslant i \leqslant n\right\}<\varepsilon\right\}\right)$.

Proof. Given in LeCam and Schwartz [1960, p. 142]. 
Lemma 4. Let $\phi^{*}:(\mathscr{M}, U) \rightarrow\left(\phi^{*}(\mathscr{M}),\|\cdot\|_{L_{1}}\right)$. The following propositions are equivalent:

(A) $\phi^{*}$ is uniformly continuous.

(B) $\mathscr{M}$ is $L_{1}$-totally bounded.

Proof. (B) $\Rightarrow(\mathrm{A})$. To prove $\phi^{*}$ is uniformly continuous, it suffices to prove that for every $\varepsilon>0$ there exist $\delta(\varepsilon)$ and $B_{1}, \ldots, B_{n}$ sets in $\mathscr{A}^{m}$ such that for every $(P, Q) \in V_{\delta(\varepsilon), m, B_{1} \ldots, B_{n}}=\left\{(\tilde{P}, \tilde{Q}): \sup \left\{\left|\tilde{P}^{m}\left(B_{i}\right)-\tilde{Q}^{m}\left(B_{i}\right)\right| ; \quad 1 \leqslant i \leqslant n\right\}<\delta(\varepsilon)\right\}$ implies $\left\|\phi^{*}(P)-\phi^{*}(Q)\right\|=\|P-Q\|_{L_{1}}<\varepsilon$.

Consider $\varepsilon / 5>0$. By $L_{1}$-total boundedness of the space $\mathscr{M}$ there exist $P_{1}, \ldots, P_{k}$ in $\mathscr{M}$ such that for every $P \in \mathscr{M}, \inf \left\{\left\|P-P_{i}\right\|_{L_{1}} ; 1 \leqslant i \leqslant k\right\}<\varepsilon$. On the other hand

$$
\left\|P_{i}-P_{j}\right\|_{L_{1}}=P_{i}\left\{x: \frac{d P_{i}}{d \mu}(x)>\frac{d P_{j}}{d \mu}(x)\right\}-P_{j}\left\{x: \frac{d P_{i}}{d \mu}(x)>\frac{d P_{j}}{d \mu}(x)\right\},
$$

where the existence of the dominating measure $\mu$ is secured by total boundedness. Consider all sets of the form $\left\{\left\{x: d P_{i}(x) / d \mu>d P_{j}(x) / d \mu\right\}\right.$ for $\left.1 \leqslant i<j \leqslant k\right\}$ and call them $B_{1}, \ldots, B_{n}$.

Consider now $V_{\varepsilon / 5,1, B_{1} \ldots, B_{n}}=\left\{(\tilde{P}, \tilde{Q}): \sup \left\{\left|\tilde{P}\left(B_{i}\right)-\tilde{Q}\left(B_{i}\right)\right| ; 1 \leqslant i \leqslant n\right\}<\varepsilon / 5\right\}$. Let $P, Q \in V_{\varepsilon / 5,1, B_{1} \ldots, B_{n}}$. We will prove $\|P-Q\|_{L_{1}}<\varepsilon$. Using the triangular inequality we get

$$
\begin{aligned}
\|P-Q\|_{L_{1}} & \leqslant\left\|P-P_{i}\right\|_{L_{1}}+\left\|P_{i}-P_{j}\right\|_{L_{1}}+\left\|P_{j}-Q\right\|<\frac{2 \varepsilon}{5}+\left\|P_{i}-P_{j}\right\|_{L_{1}} \\
& \leqslant \frac{4 \varepsilon}{5}+\sup \left\{\left|P\left(B_{i}\right)-Q\left(B_{i}\right)\right| ; 1 \leqslant i \leqslant n\right\}<\varepsilon .
\end{aligned}
$$

(A) $\Rightarrow$ (B). To prove now that $\mathscr{M}$ is $L_{1}$-totally bounded, it suffices to prove that for every $\varepsilon>0$ there exist $P_{1}, \ldots, P_{l}$ in $\mathscr{M}$ such that $\inf \left\{\left\|P-P_{i}\right\| ; 1 \leqslant i \leqslant l\right\}<\varepsilon$ for every $P$ in $\mathscr{M}$.

Fix $\varepsilon>0$. Since $\phi^{*}$ is uniformly continuous for that $\varepsilon$ there exist $\delta(\varepsilon), m(\varepsilon)$ and $B_{1}, \ldots, B_{n}$, in $\mathscr{A}^{m(\varepsilon)}$ such that if $(P, Q) \in V_{\delta(\varepsilon), m(\varepsilon), B_{1} \ldots, B_{n}}$, then $\left\|\phi^{*}(P)-\phi^{*}(Q)\right\|=$ $\|P-Q\|_{L_{1}}<\varepsilon$. By Lemma $3, U$ is precompact so for $V_{\delta(\varepsilon), m(\varepsilon), B_{1}, \ldots, B_{n}}$ there exist $P_{1}, \ldots, P_{1}$ such that $\mathscr{M}=\bigcup_{k=1}^{\prime}\left\{P: \sup \left\{\left|P^{m(\varepsilon)}\left(B_{i}\right)-P_{k}^{m(\varepsilon)}\left(B_{i}\right)\right| ; 1 \leqslant i \leqslant n\right\}<\delta(\varepsilon)\right\}$. From this it follows that $\inf \left\{\left\|P-P_{i}\right\|_{L_{1}} ; 1 \leqslant i \leqslant l\right\}<\varepsilon$ for every $P \in \mathscr{M}$.

Lemma 5. Assume now that $\mathscr{M}$ is $L_{1}$-separable, $\phi^{*}:(\mathscr{M}, U) \rightarrow\left(\phi^{*}(\mathscr{M}),\|\cdot\|_{L_{1}}\right)$, and $\mu$ is a dominating measure existing by $L_{1}$-separability assumption. The following propositions are equivalent:

(B) $\mathscr{M}$ is $L_{1}$-totally bounded.

(C) (a) There exists a uniformly consistent estimator for $\phi^{*}(P)$ with values in $\phi^{*}(\mathscr{M})$, (b) for every $\varepsilon>0$ there exists a $\delta(\varepsilon)>0$ such that if $\mu(A)<\delta(\varepsilon)$, then $\sup \{P(A) ; P \in \mathscr{M}\}<\varepsilon$.

Proof. (B) $\Rightarrow(\mathrm{C})$. We will first prove (b). Fix $\varepsilon>0$. For $\varepsilon / 2$ there exist $P_{1}, \ldots, P_{n}$ in $\mathscr{M}$ such that $\inf \left\{\left\|P-P_{i}\right\|_{L_{1}} ; 1 \leqslant i \leqslant n\right\}<\varepsilon / 2$ for every $P$ in $\mathscr{M}$. By absolute continuity of $P_{1}, \ldots, P_{n}$ with respect to $\mu$ there is a $\delta(\varepsilon)>0$ such that if $\mu(A)<\delta(\varepsilon)$, then $\sup \left\{P_{i}(A) ; 1 \leqslant i \leqslant n\right\}<\varepsilon / 2$. Consider now $P \in \mathscr{M}$. Then

$$
P(A)=P(A)-P_{i}(A)+P_{i}(A) \leqslant\left\|P-P_{i}\right\|_{L_{1}}+P_{i}(A)<\varepsilon / 2+\varepsilon / 2=\varepsilon,
$$


where $P_{i}$ is the center of the $L_{1}$-ball of radius $\varepsilon / 2$ containing $P$ and this holds for every $A \in \mathscr{A}$ such that $\mu(A)<\delta(\varepsilon)$ and for every $P$ in $\mathscr{M}$.

We now prove (a) by constructing a uniformly consistent minimum distance estimator.

Let $a_{n}$ be a sequence of numbers such that $a_{1} \geqslant a_{2} \geqslant \cdots \geqslant a_{n} \geqslant \cdots \geqslant 0$ tending to 0 . Since $\mathscr{M}$ is $L_{1}$-totally bounded for every $a_{n}$, there is an $a_{n}$-dense subset of measures $P_{1}, \ldots, P_{N\left(a_{n}\right)}$ in $\mathscr{M}$. Let

$$
\mathscr{F}_{a_{n}}=\left\{\left\{x: \frac{d P_{i}}{d \mu}(x)>\frac{d P_{j}}{d \mu}(x)\right\}, 1 \leqslant i<j \leqslant N\left(a_{n}\right)\right\} .
$$

By applying the triangular inequality, we have then that for every $P, Q$ in $\mathscr{M}$, $\|P-Q\|_{L_{1}} \leqslant 4 a_{n}+2 \sup \left\{|P(A)-Q(A)| ; A \in \mathscr{F}_{a_{n}}\right\}$.

Let $X_{1}, \ldots, X_{k}$ be independent identically distributed observations from $P \in \mathscr{M}$ and $\mu_{k}(A)=\frac{1}{k} \sum_{i=1}^{k} I_{A}\left(X_{i}\right)$ the empirical measure indexed by $A \in \mathscr{A}$. Define an estimator

$$
\begin{aligned}
\hat{P}_{k, n} & : \sup \left\{\left|\mu_{k}(A)-\hat{P}_{k, n}(A)\right| ; A \in \mathscr{F}_{a_{n}}\right\} \\
& =\inf \left\{\sup \left\{\left|\mu_{k}(A)-Q(A)\right| ; A \in \mathscr{F}_{a_{n}}\right\} ; Q \in \mathscr{M}\right\}
\end{aligned}
$$

(without loss of generality we can assume the infimum is achieved). For fixed $a_{n}$, by the law of large numbers, $\sup \left\{\left|\mu_{k}(A)-\hat{P}_{k, n}(A)\right| ; A \in \mathscr{F}_{a_{n}}\right\} \leqslant \sup \left\{\left|\mu_{k}(A)-P(A)\right|\right.$; $\left.A \in \mathscr{F}_{a_{n}}\right\} \rightarrow 0$ as $k$ tends to infinity. So there is a $K\left(a_{n}\right)$ such that

$$
\sup \left\{\left|\mu_{k}(A)-\hat{P}_{k, n}(A)\right| ; A \in \mathscr{F}_{a_{n}}\right\} \leqslant a_{n} \text { for } K \geqslant K\left(a_{n}\right) .
$$

Repeating the same construction for $\mathscr{F}_{a_{n+1}}$, there exists $K\left(a_{n+1}\right)$ such that $\sup \left\{\left|\mu_{k}(A)-\hat{P}_{k, n+1}(A)\right| ; A \in \mathscr{F}_{a_{n+1}}\right\}<a_{n+1}$ for $K \geqslant K\left(a_{n+1}\right)$. Let $K\left(a_{n}, a_{n+1}\right)=$ $\max \left\{K\left(a_{n}\right), K\left(a_{n+1}\right)\right\}$ and define

$$
\hat{P}_{k}= \begin{cases}\hat{P}_{k, n} & \text { for } K \leqslant K\left(a_{n}, a_{n+1}\right), \\ \hat{P}_{k, n+1} & \text { for } K\left(a_{n}, a_{n+1}\right)<K \leqslant K\left(a_{n+1}, a_{n+2}\right) .\end{cases}
$$

We claim that $\left\|\hat{P}_{k}-P\right\|_{L_{1}} \rightarrow 0$ as $k$ tends to infinity in $P^{k}$-probability. By construction,

$$
\begin{aligned}
\left\|\hat{P}_{k}-P\right\|_{L_{1}} & \leqslant 4 a_{n}+2 \sup \left\{\left|\hat{P}_{k}(A)-P(A)\right| ; A \in \mathscr{F}_{a_{n}}\right\} \\
& \leqslant 4 a_{n}+2 \sup \left\{\left|\mu_{k}(A)-\hat{P}_{k}(A)\right| ; A \in \mathscr{F}_{a_{n}}\right\} \leqslant 5 a_{n}
\end{aligned}
$$

for $K \geqslant K\left(a_{n}, a_{n+1}\right)$ with high probability. Q.E.D.

$(C) \Rightarrow(B)$. By Lemma 4 it is enough to prove $(C) \Rightarrow(A)$, i.e. we will prove that $\phi^{*}$ is uniformly continuous.

By assumption there is a uniformly consistent estimator $T_{n}$ of $\phi^{*}(P)$ taking values in $\phi^{*}(\mathscr{M})$. So for every $n$, there is $\hat{P}_{n}$ in $\mathscr{M}$ such that $T_{n}=\phi^{*}\left(\hat{P}_{n}\right)$. By uniform consistency we will then have

$$
\sup \left\{E_{P^{\prime \prime}}\left\|T_{n}-\phi^{*}(P)\right\| ; P \in \mathscr{M}\right\}=\sup \left\{E_{P^{\prime \prime}}\left\|\hat{P}_{n}-P\right\|_{L_{1}} ; P \in \mathscr{M}\right\} \rightarrow 0
$$

as $n$ tends to infinity. 
Fix $\varepsilon>0$ and consider

$$
\begin{aligned}
\left\|\phi^{*}(P)-\phi^{*}(Q)\right\| & =\|P-Q\|_{L_{1}} \\
& \leqslant\left\|P-E_{P^{n}} \hat{P}_{n}\right\|_{L_{1}}+\left\|E_{P^{n}} \hat{P}_{n}-E_{Q^{n}} \hat{P}_{n}\right\|_{L_{1}}+\left\|E_{Q^{n}} \hat{P}_{n}-Q\right\|_{L_{1}} \\
& \leqslant E_{P^{n}}\left\|P-\hat{P}_{n}\right\|_{L_{1}}+\left\|E_{P^{n}} \hat{P}_{n}-E_{Q^{n}} \hat{P}_{n}\right\|_{L_{1}}+E_{Q^{n}}\left\|\hat{P}_{n}-Q\right\|_{L_{1}} \\
& \leqslant 2 \varepsilon+\left\|E_{P^{n}} \hat{P}_{n}-E_{Q^{n}} \hat{P}_{n}\right\|_{L_{1}}
\end{aligned}
$$

for all $n \geqslant n(\varepsilon)$ by (4). Let $n=n(\varepsilon)$ for the rest of the proof.

Observe now that $\hat{P}_{n}$ takes values in the space $\mathscr{M}$ which is $L_{1}$-separable. By separability there exists a dominating measure $\mu$. Also strong measurability is equivalent to weak measurability (so we do not have any measurability problems) and there exist sets $A_{i}^{n}$ in $\mathscr{A}^{n}, i=1,2, \ldots$, such that $\left\|\hat{P}_{n}-\sum_{i=1}^{\infty} I_{A_{i}^{n}} P_{i}\right\|_{L_{1}}<\varepsilon$ a.e. $\mu^{n}$, with $\left\{P_{i}\right\}, 1 \leqslant i \leqslant n$, being the countable dense subset of $\mathscr{M}$.

So we finally have

$$
\left\|\phi^{*}(P)-\phi^{*}(Q)\right\| \leqslant 4 \varepsilon+\left\|E_{P^{n}} \sum_{i=1}^{\infty} I_{A_{i}^{n}} P_{i}-E_{Q^{n}} \sum_{i=1}^{\infty} I_{A_{i}^{n}} P_{i}\right\| .
$$

As we know, $\sum_{i=1}^{\infty} I_{A_{i}^{n}} P_{i}=\lim _{k \rightarrow \infty} \sum_{i=1}^{k} I_{A_{i}^{n}} P_{i}$ in $L_{1}, \mu^{n}$ a.s. and the convergence is almost uniform $\mu^{n}$, so for $\delta(\varepsilon)>0$ there exists $E_{\varepsilon}$ in $\mathscr{A}^{n}$ such that $\mu^{n}\left(E_{\varepsilon}\right)<\delta(\varepsilon)$ and for $K=K(\varepsilon)$ big enough $\left\|\sum_{i=1}^{\infty} I_{A_{i}^{n}} P_{i}-\sum_{i=1}^{k} I_{A_{i}^{n}} P_{i}\right\|_{L_{1}}<\varepsilon$ for all $x$ in $X^{n}-E_{\varepsilon}$.

We will try now to evaluate the right-hand side of $(5)$ :

$$
\begin{aligned}
& \left\|E_{P^{n}} \sum_{i=1}^{\infty} I_{A_{i}^{n}} P_{i}-E_{P^{n}} \sum_{i=1}^{k} I_{A_{i}^{n}} P_{i}\right\| \\
& \quad=\left\|\int_{E_{\varepsilon}}\left(\sum_{i=1}^{\infty} I_{A_{i}^{n}} P_{i}-\sum_{i=1}^{k} I_{A_{i}^{n}} P_{i}\right) d P^{n}+\int_{\mathscr{X}^{n}-E_{\varepsilon}}\left(\sum_{i=1}^{\infty} I_{A_{i}^{n}} P_{i}-\sum_{i=1}^{k} I_{A_{i}^{n}} P_{i}\right) d P^{n}\right\|_{L_{1}} \\
& \quad \leqslant 2 P^{n}\left(E_{\varepsilon}\right)+\varepsilon \cdot P^{n}\left(\mathscr{X}^{n}-E_{\varepsilon}\right) \leqslant 3 \varepsilon .
\end{aligned}
$$

Repeating the same type of calculation for $E_{Q^{n}} \sum_{i=1}^{\infty} I_{A_{i}^{n}} P_{i}$ and replacing in (5), we get

$$
\begin{aligned}
\left\|\phi^{*}(P)-\phi^{*}(Q)\right\| & \leqslant 10 \varepsilon+\left\|E_{P^{n}} \sum_{i=1}^{k} I_{A_{i}^{n}} P_{i}-E_{Q^{n}} \sum_{i=1}^{k} I_{A_{i}^{n}} P_{i}\right\|_{L_{1}} \\
& =10 \varepsilon+\sup \left\{\left|\sum_{i=1}^{k}\left(P^{n}\left(A_{i}^{n}\right)-Q^{n}\left(A_{i}^{n}\right)\right) \cdot P_{i}(A)\right| ; A \in \mathscr{A}\right\} \\
& \leqslant 10 \varepsilon+K \cdot \sup \left\{\left|P^{n}\left(A_{i}^{n}\right)-Q^{n}\left(A_{i}^{n}\right)\right| ; 1 \leqslant i \leqslant k\right\} . \text { Q.E.D. }
\end{aligned}
$$

From Lemmas 4 and 5 we have the following

THEOREM 2. Let $\mathscr{M}$ be an $L_{1}$-separable family of measures, $\phi^{*}:(\mathscr{M}, U) \rightarrow$ $\left(\phi^{*}(\mathscr{M}),\|\cdot\|_{L_{1}}\right)$ and $\mu$ a dominating measure. The following positions are equivalent.

(A) $\phi^{*}$ is uniformly continuous.

(B) $\mathscr{M}$ is $L_{1}$-totally bounded. 
(C) (a) There exists a uniformly consistent estimator for $\phi^{*}(P)$ with values in $\phi^{*}(\mathscr{M})$, (b) for every $\varepsilon>0$ there exists a $\delta(\varepsilon)>0$ such that for every $A$ in $\mathscr{A}$ : $\mu(A)<\delta(\varepsilon), \sup \{P(A) ; P \in \mathscr{M}\}<\varepsilon$.

REMARK. This theorem shows that in the case that $\mathscr{M}$ is uniformly dominated every uniformly consistent estimator in $L_{1}$ can be achieved through minimum distance. On the other hand the condition of uniform domination is not a necessary condition for the existence of uniformly consistent estimates in $L_{1}$ as the example of families of densities satisfying the Hoeffding-Wolfowitz [1958] condition shows.

Acknowledgment. This work is part of the author's Ph.D. Thesis at the University of California, Berkeley, written under the supervision of Professor Lucien M. LeCam whose patience and suggestions are gratefully acknowledged and appreciated.

\section{REFERENCES}

G. Choquet [1969], Lectures on analysis, Vol. I, Chapter 2, §5, Benjamin, New York.

E. Hille and R. Phillips [1957], Functional analysis and semi-groups, Amer. Math. Soc. Colloq. Publ., vol. 31, Amer. Math. Soc., Providence, R. I.

W. Hoeffding and J. Wolfowitz [1958], Distinguishability of sets of distributions, Ann. Statist. 29, $700-718$.

C. Kraft [1955], Some conditions for consistency and uniform consistency of statistical procedures, Univ. of Calif. Publ. in Statist. 2, 125-142.

L. M. LeCam and L. Schwartz [1960], A necessary and sufficient condition for the existence of consistent estimates, Ann. Statist. 31, 140-150.

R. Moché [1977], Thèse de Doctorat d'Etat, Université de Lille, France.

J. Pfanzagl [1968], On the existence of consistent estimates and tests, Z. Wahrsch. Verw. Gebiete 10, $43-62$.

Y. G. Yatracos [1983], Uniformly consistent estimates and rates of convergence via minimum distance methods, Ph.D. Thesis, Univ. of California, Berkeley, Calif.

Department of Statistics, University of California, Berkeley, California 94720

Current address: Department of Statistics, Hill Center for the Mathematical Sciences, Busch Campus. Rutgers University, New Brunswick, New Jersey 08903 